cemoti $\begin{aligned} & \text { Cahiers d'études sur la Méditerranée } \\ & \text { orientale et le monde turco-iranien }\end{aligned}$

22 | 1996

Arabes et Iraniens

\title{
Sayyida Zaynab, Banlieue de Damas ou nouvelle ville sainte chiite?
}

Sabrina MERVIN

\section{(2) OpenEdition}

Édition électronique

URL : https://journals.openedition.org/cemoti/138

DOI : $10.4000 /$ cemoti. 138

ISSN : 1777-5396

Éditeur

AFEMOTI

Édition imprimée

Date de publication : 1 juin 1996

ISSN : 0764-9878

Référence électronique

Sabrina MERVIN, «Sayyida Zaynab, Banlieue de Damas ou nouvelle ville sainte chiite ? », Cahiers d'études sur la Méditerranée orientale et le monde turco-iranien [En ligne], 22 | 1996, mis en ligne le 04 mars 2005, consulté le 20 juin 2022. URL : http://journals.openedition.org/cemoti/138 ; DOI : https:// doi.org/10.4000/cemoti. 138

Ce document a été généré automatiquement le 20 juin 2022.

Tous droits réservés 


\title{
Sayyida Zaynab, Banlieue de Damas ou nouvelle ville sainte chiite?
}

\author{
Sabrina MERVIN
}

De chaque côté de la route défilent des murs peints d'un fond bleu sur lesquels des sentences sont calligraphiées : louanges de Bâsil al-Asad, le défunt dauphin du président syrien. Ayant trouvé la mort dans un accident d'automobile ${ }^{1}$, il est maintenant un "martyr". Voici son portrait en cavalier émérite ; le voilà en officier exemplaire, le visage barré de ses éternelles lunettes noires ; plus loin, le dessin d'un oeil - celui de la Syrie - qui verse des larmes de chagrin... Ces portraits jalonnent la route du Sud, qui relie Damas à la Jordanie. C'est aussi la route qui mène à Sayyida Zaynab, où plus d'un million de pèlerins passent chaque année pour aller pleurer d'autres martyrs, les ahl al-bayt, les membres de la famille du Prophète.

2 Ils viennent d'Iran, d'Irak, du Golfe arabo-persique, du Pakistan, d'Afghanistan, mais aussi du Liban voisin et de Syrie. Bien que l'on ne puisse obtenir de pourcentage précis, en grande majorité, les pèlerins sont chiites duodécimains².

3 Rendre une visite pieuse au mausolée de Zaynab, fille de l'imâm 'Alî et petite-fille du Prophète, revient pour eux à accomplir "le pèlerinage des pauvres" (hâgg al-fuqarâ'), lorsqu'ils ne peuvent aller ni à La Mecque, ni dans les sanctuaires d'Irak, Najaf, Karbalâ', Samarrâ' et Kâzimayn ${ }^{3}$. Ils viennent rendre grâce à "la Dame" (al-sitt), lui demander son concours, pleurer sur ses malheurs et ceux de la Famille sacrée, avant d'aller se recueillir sur la tombe d'Ali Shariati ${ }^{4}$, sise dans le cimetière près du mausolée.

4 Le tombeau de la sainte fut longtemps fréquenté autant par les sunnites que par les chiites. Dans le récit de ses voyages, l'andalou Ibn Gubayr (m.1217), rapporte que les chiites occupent dans la région une place extraordinaire et qu'ils sont plus nombreux que les sunnites; il raconte s'être rendu au mausolée de Sayyida Zaynab pour y passer la nuit et recueillir la baraka de la sainte ${ }^{5}$. Au XIV ${ }^{\mathrm{e}}$ siècle, les chiites ne constituent probablement plus la majorité des musulmans de Syrie; ils continuent toutefois à être présents à Damas ${ }^{6}$. A cette époque, Ibn Battûta évoque le tombeau de Zaynab, il est flanqué d'une mosquée et doté de legs pieux 7 . D'autres auteurs mentionnent ensuite le mausolée; ils ne s'accordent pas tous à reconnaître que c'est celui de Zaynab, fille de 
'Alî ${ }^{8}$. Le soufi 'Abd al-Ganî al-Nâbulsî (m. 1731) fait état d'une tombe abritée par une immense coupole, jouxtant une mosquée et un bassin, dont "on dit" qu'elle est celle de Sitt Zaynab. Mais pour lui, Zaynab est enterrée au Caire... ${ }^{9}$.

5 Théoriquement, le mausolée demeura un centre de dévotion pour tous les musulmans mais, pratiquement, il se "chiitisa" peu à peu à partir de la fin du siècle dernier jusqu'à aujourd'hui où le processus est encore observable. A cela ont concouru deux éléments essentiels : le rite de 'Asûrâ', qui y est chaque année célébré par les chiites ${ }^{10}$ et la venue massive de pèlerins chiites étrangers, surtout iraniens. Ainsi, aux yeux de la majorité sunnite de Syrie, le mausolée finit par devenir doublement étranger. En premier lieu à cause des pratiques cultuelles chiites distinctes de celles du sunnisme. Ainsi de 'Asûrâ' mais aussi du culte rendu à la sainte qui, comme les visites pieuses faites aux imâm-s $\mathbf{s}^{11}$, se distingue des pratiques sunnites jusque dans ses manifestations les plus extérieures : les chiites, dès l'entrée dans le mausolée, se mettent à se lamenter et à verser des larmes... Le second facteur d'extranéité vient du fait que la majorité des pèlerins sont des Iraniens, c'est-à-dire non arabes, 'agam, ce qui se voit, ne serait-ce que par un simple regard au costume des femmes et ce qui s'entend, à l'écoute de leur langue ou de leur acccent.

6 Les groupes de pèlerins iraniens sont pris en charge par une institution syro-iranienne qui organise leurs pérégrinations à Damas et en Syrie, les transportant en car d'un lieu saint à un autre. A Damas, le cimetière de Bâb Sagîr, où sont enterrés de nombreux ahl al-bayt et autres martyrs de Karbalâ', la mosquée des Omeyyades, que l'on dit renfermer la tête de l'imâm Husayn, et le mausolée de sayyida Ruqayya, dans le quartier al-'Amâra, au coeur de la vieille ville, qui fut récemment rénové à grands frais dans le style iranien. A Alep, un mausolée rénové grâce à des fonds iraniens abrite un rocher où la tête de Husayn aurait reposé et où une goutte de son sang aurait coulé; un autre recèlerait le tombeau de Muhsin, fils de Husayn, décédé avant même de naître. Il y a quelques autres sanctuaires à Raqqa (qui est en cours de rénovation grâce à des fonds iraniens), Homs, Hama, 'Adrâ' ...12.

7 Sayyida Zaynab reste cependant le pôle d'attraction des pèlerins. Située à sept kilomètres de la capitale, l'agglomération a sa propre vie économique et ses infrastructures touristiques sont en plein développement. Pour compléter les équipements existants, deux hôtels d'une capacité de 600 chambres (l'un de catégorie moyenne, l'autre de luxe) sont en construction au nord-ouest du mausolée ; des restaurants et différents services sont prévus, à l'intérieur et autour de ces hôtels. Sayyida Zaynab devient un centre économique et touristique, ce qui est largement le fruit des activités commerciales déployées par un riche homme d'affaires syrien, le chiite Sâ'ib Nahhâs. Non seulement une filiale de son groupe est le promoteur de ce projet hôtelier, mais sa société Transtour est la seule compagnie autorisée à prendre les pèlerins chiites en charge et il est le fondateur et le président d'une "Commission de coordination économique entre la Syrie et l'Iran"13.

8 L'endroit a été véritablement bouleversé depuis les années 50. Râwiya ou Qabr al-Sitt, comme on appelait alors le village, comptait environ deux cents habitants, des agriculteurs. Il connut deux immigrations massives : en 1949, celle de réfugiés palestiniens et en 1967, celle de réfugiés du Golan. Puis des habitants du village de Fû́ ${ }^{\prime}{ }^{14}$ vinrent $s^{\prime} y$ installer. Les Irakiens affluèrent à partir des années 70 , et surtout pendant la guerre irano-irakienne; ils furent bientôt rejoints par des ressortissants 
chiites d'autres pays. Ainsi, la population de Sayyida Zaynab passa de 800 habitants en $1960^{15}$ à environ 100000 habitants aujourd'hui ${ }^{16}$.

9 Quant au mausolée, il a été entièrement rénové dans les années 50 , grâce aux efforts déployés par un comité formé ad hoc en 1951, regroupant des notables chiites de Syrie et un Irakien, al-hâgg Bahbahânî. Ils menèrent campagne auprès de leurs coreligionnaires iraniens afin de réunir les fonds nécessaires à la restauration du mausolée. Ce qui fut fait. Des chèques furent envoyés de Qumm et d'ailleurs à Muhsin al-Amîn, alors le marga ${ }^{177}$ des chiites de Syrie, et les travaux commencèrent ${ }^{18}$. Ce n'était ni la première, ni la dernière fois que le mausolée de sayyida Zaynab allait bénéficier des largesses de particuliers. En 1935, des notables syriens de la famille Nizâm firent rénover le bâtiment de l'entrée ouest ; en 1950, un commerçant pakistanais, Muhammad 'Alî Habîb, suite à la guérison prodigieuse de son fils, offrit les précieuses grilles d'argent qui servent d'écrin au tombeau. Puis un commerçant du Golfe paya la couverture en mosaïque des deux minarets du sanctuaire. Le tombeau de la sainte est un "cadeau du peuple iranien" offert en 1954. Six ans plus tard, un commerçant iranien fit don d'une porte dorée, que l'on mit à l'entrée ouest du sanctuaire ${ }^{19}$.

10 Le mausolée profitant d'initiatives privées, la communauté chiite de Syrie garde une emprise sur lui, à travers la famille Murtadâ qui gère ses waqfs depuis le $\mathrm{XIV}^{\mathrm{e}}$ siècle $^{20}$. L'État syrien a avalisé la gestion du waqf par cette famille, à qui échoit également celle des waqfs de mausolées de ahl al-bayt à Damas. La situation est tout autre pour le mausolée de Sayyida Ruqayya, situé dans la vieille ville de Damas. En effet, sa rénovation et son agrandissement ont été entièrement et directement financés par le gouvernement iranien, en coopération avec le ministère syrien des waqfs. Pour ce faire, une partie d'un ancien quartier a été rasé, ce qui provoqua la grogne muette des habitants de la vieille ville. Il y eut, en fait, un accord réalisé d'État à État, par-dessus la communauté chiite de Syrie, qui a en quelque sorte été dépossédée du mausolée.

11 Hormis les anciens habitants de Fû́a, la population syrienne de Sayyida Zaynab est sunnite ; les chiites, eux, vivent à Damas, surtout dans leurs anciens quartiers de Kharâb dit "al-Amîn", Jûra et Sâlihiyya. La ville a été investie par d'autres communautés chiites que celle de Syrie : de nombreux réfugiés irakiens y résident à côté de Pakistanais, d'Afghans, d'Iraniens, etc. Ils ont eu les coudées franches pour y installer leurs lieux de culte et leurs institutions religieuses ou caritatives et il y a maintenant à Sayyida Zaynab une demi-douzaine de Husayniyyât, lieux consacrés aux commémorations du martyre de Husayn, sans compter les associations culturelles ou de bienfaisance. C'est le seul endroit en Syrie où l'appel à la prière est effectué selon le rite ga'farite.

12 La ville est devenue un foyer de savoir du chiisme duodécimain et certains aspirent à ce qu'elle parvienne à remplacer Nagaf, qui semble dans la dernière phase de son déclin. En effet, Sayyida Zaynab renferme actuellement neuf écoles religieuses (hawzas) fréquentées, dit-on, par plus de mille étudiants. Foyer de savoir, pépinière de clercs et pôle de sacré grâce à son mausolée, Sayyida Zaynab serait alors le centre du chiisme arabe susceptible de concurrencer Qumm, abritant le mausolée d'une autre sainte chiite, Fâtima, soeur du huitième imâm, Ridâ, ce dont les centres d'enseignement chiites du Liban sont incapables, puisqu'ils ne recèlent pas de lieu saint. La Syrie ne serait alors que la terre d'accueil de ce centre car il n'y a plus de tradition d'enseignement chiite depuis que celle d'Alep s'est éteinte au XVI $\mathrm{e}^{\mathrm{e}}$ siècle, et il n'y a pas de grand mugtahid syrien ${ }^{21}$. Muhsin al-Amîn, qui vint s'installer à Damas en 1901, à la 
demande de la communauté chiite, en fut le chef spirituel jusqu'en 1952 : il était originaire du Gabal 'Amil (Sud-Liban), tout comme ses successeurs Husayn Makkî puis le fils de celui-ci, 'Alî Makkî.

13 Al-Hawza al-Zaynabiyya, la première école religieuse de Sayyida Zaynab, fut ouverte en 1973, à l'initiative du mugtahid irakien Sayyid Hasan Sîrâzî (1934-1980). Opposant au régime de Saddâm Husayn, il vivait à Beyrouth d'où il se rendait régulièrement en Syrie, et en particulier à Sayyida Zaynab, pour prononcer des conférences et nouer des contacts avec des personnalités politiques. Lorsqu'un grand nombre d'étudiants en sciences religieuses de Nagaf se firent expulser d'Irak, il partit chercher les fonds nécessaires dans les pays du Golfe et, grâce à ses appuis syriens, fonda une hawza à Sayyida Zaynab, avec un "foyer" pour loger enseignants et élèves. Il eut même l'accord des autorités syriennes pour que l'inscription à l'école permette aux étudiants d'obtenir une carte de séjour ${ }^{22}$. Depuis la disparition du fondateur, assassiné à Beyrouth par des agents irakiens, c'est son frère l'ayatollah Muhammad al-Sîrâzî qui supervise les activités de l'école, de Qumm où il est lui-même réfugiée ${ }^{23}$. Elle renferme aujourd'hui 220 étudiants.

14 Une dizaine d'années plus tard, c'est une hawza d'obédience directement iranienne qui vit le jour, Hawzat al-imâm al-Khumaynî. Elle fut créée en 1982 à l'initiative de l'agent (wakîl) ${ }^{24}$ de Khumaynî, qui est aujourd'hui celui de Khamenei, et regroupe aujourd'hui environ 150 étudiants: des Syriens, des Irakiens et des Iraniens, mais aussi des ressortissants de différents pays d'Afrique (Sierra Leone, Côte d'Ivoire, Guinée, Tanzanie, etc.) et d'Asie (Pakistan, Afghanistan, Inde, Indonésie). Ses enseignants, eux aussi de différentes nationalités, ont en général étudié à Qumm ${ }^{25}$. D'autres écoles ont suivi, avec de moindres capacités d'accueil et moins d'étudiants inscrits ; moins organisées, aussi, et souvent éphémères. Rien d'étonnant à cela. D'abord, le phénomène est dû à la labilité qui caractérise en général les écoles religieuses chiites où il n'y a pas de bureaucratie centralisée et qui fonctionnent selon le même schéma traditionnel ${ }^{26}$ : un clerc réputé pour son savoir prend l'initiative d'ouvrir une école, son charisme attire à lui à la fois les riches donateurs et les disciples nécessaires à la concrétisation du projet. Si le clerc en question quitte l'endroit pour aller s'installer ailleurs, ses disciples le suivent; s'il vient à mourir, les étudiants se dispersent dans d'autres écoles. Car c'est avant tout l'enseignement d'un mujtahid qui est recherché par ses disciples qui l'admirent pour l'étendue de sa science, l'habileté de sa méthode mais aussi pour sa piété et ses qualités morales. La souplesse de l'organisation qui est réduite au minimum, la liberté dont jouissent les étudiants de choisir leurs maîtres et les matières qu'ils veulent approfondir, l'absence d'examen contrôlant l'acquisition des connaisances font que ce système repose entièrement sur les individus, mujtahid-s et étudiants ${ }^{27}$. Les cours du dernier cycle d'étude, appelé baht al-hârig (étude "hors" des livres, parce qu'on ne les utilise plus) consistent en l'examen d'une question précise, d'abord par le maître, puis par les étudiants qui sont invités à défendre leur avis en explicitant leur argumentation. Ce qui favorise l'émulation entre les étudiants et les entraîne à la "déduction de jugement légaux" (istinbât al-ahkâm al-sar'iyya), c'est-à-dire leur confère la compétence d'ijitihâd.

15 Ce système traditionnel était appliqué dans les écoles de Nagaf, malgré la tentative de réforme opérée à la fin des années cinquante par un aréopage de mugtahid-s désireux de le rationaliser et de le moderniser ${ }^{28}$. C'est au sein de ce système traditionnel que Abû al-Qâsim al-Khû'î (m. 1992), surnommé "le chef de file de l'école" (zâîm al-hawza 
al-'ilmiyya ${ }^{29}$ avait formé des dizaines de mugtahid-s et s'était imposé comme marga ${ }^{4}$, référence à imiter en matière de préceptes religieux ${ }^{30}$. La crédibilité en matière de sciences religieuses et le prestige, dans tout le monde chiite, de grands mugtahid-s tels Khû'î rejaillissaient sur toutes les écoles de Nagaf, considérée comme le centre, avec Qumm, du savoir chiite. Aujourd'hui, bon nombre de ses mugtahid-s les plus réputés ont fui Nagaf qui ne peut plus remplir ce rôle. Sayyida Zaynab pourrait-elle prendre le relais?

Nous avons vu que les écoles de Sayyida Zaynab - le caractère éphémère de certaine et la souplesse de l'organisation de toutes - relevaient du système d'enseignement religieux traditionnel chiite. D'autres éléments doivent cependant être pris en compte pour expliquer ces deux phénomènes. Mentionnons en premier lieu la précarité de la situation des acteurs qui jouent un rôle dans ces écoles, à savoir à la fois les étudiants et les enseignants, qui sont en majorité des étrangers en Syrie ${ }^{31}$. Certains sont des réfugiés politiques, même s'ils n'en ont pas officiellement le statut ; beaucoup sont là en "transit", nourrissant le projet soit de retourner chez eux, soit d'émigrer ailleurs, en Europe par exemple ou bien en Iran. Autre facteur de précarité : tous sont tributaires du ou des riches donateurs permettant à l'école de fonctionner, c'est-à-dire d'une personne susceptible d'investir son capital financier dans une autre école ou bien dans une autre institution caritative ou religieuse, selon les opportunités du marché des biens de salut. Cela, afin de maximiser ses intérêts en capital symbolique. Et ce, même si ce financement se fait par l'intermédiaire d'un marga local ou de son agent qui reverse dans la caisse d'une école les impôts canoniques perçus auprès de ces riches donateurs. Il existe cependant une entorse politique au libéralisme de cette économie religieuse : l'État iranien. Celui-ci est, en effet, susceptible de se substituer au riche donateur, soit directement, soit par l'intermédiaire de l'agent du marga' qu'il a officiellement désigné, aujourd'hui 'Alî Khamenei. Dans ce cas, le sort de l'école est étroitement lié aux vicissitudes politiques.

Toutes ces questions rejoignent l'épineux problème de la marga'iyya dans ses développements récents, d'une part, et locaux, d'autre part. Cette institution chiite est en crise depuis la mort de l'ayatollah Khû'î parce qu'après lui, aucun mugtahid ne parvint à réaliser l'unanimité pour être désigné par ses pairs marga a alâ : référence suprême et chef spirituel reconnu et suivi dans tout le monde chiite. D'où la naissance de deux grands courants : le premier obtempère au choix officiel de l'Iran, qui désigne "Alî Khamenei, le second suit "l'école" de Khû'î avec ses disciples formés à Nagaf (et néanmoins d'origine iranienne) : ainsi, notamment, de 'Alî al-Sîstânî, toujours nagafite, et de Muhammad al-Rûhânî, qui réside en Iran. Dans cette situation incertaine, des clercs invoquant l'absence d'authentique marga'iyya a âa, y renoncent et s'en tiennent à l'idée d'une marga'iyya plurielle. Et de s'autoriser du témoignage de l'histoire qui atteste de cette pluralité. Ainsi se proposent-ils comme autant de marga-s locaux à rayonnement régional.

Qu'en est-il à Sayyida Zaynab et en Syrie?

19 Il faut, sur ce point, distinguer la banlieue de Damas du reste du pays car, comme nous l'avons souligné, la communauté chiite syrienne compte très peu de représentants à Sayyida Zaynab. Or, elle a son propre marga ${ }^{c 32}$, 'Alî Makkî, un clerc originaire du SudLiban qui a pris la succession de son père dans cette fonction et se rattacherait plutôt aux disciples de Khû'î qu'au successeur de Khumayni. Il n'est pas partie prenante dans la course opposant les concurrents à la marga iyya "locale" ${ }^{33}$ car il ne dispose ni de la 
renommée de savant nécessaire ni d'un forum où diffuser ses idées, comme le font ses homologues libanais. En revanche, il y a dans le Liban voisin des candidats à la marga iyya locale, formés à Nagaf : Muhammad Taqî al-Faqîh, Muhammad Husayn Fadl Allâh et Muhammad Mahdî Sams al-Dîn. Le nom du premier a été même avancé pour la désignation à la marga'iyya alâ. Il remplit en effet certaines conditions exigées, comme une science qui s'étale dans de nombreuses publications en matière de fiqh, une piété et un dédain pour les choses de ce monde que personne ne saurait lui nier. Muhammad Taqî al-Faqîh vit en effet modestement, retiré à Tyr, où il s'occupe des fidèles sans se pencher sur les questions politiques qui mobilisent pourtant la plupart de ses pairs libanais. Mais ce quiétiste n'a pas eu l'intense activité d'enseignement qu'on attend du candidat à la marga'iyya suprême et il se contente d'un magistère restreint. Les deux candidats suivants ont plus d'ambition mondaine. Muhammad Husayn Fadl Allâh, guide spirituel d'un Hezbollah toujours présent sur le terrain de la "résistance islamique", se comporte comme un marga' local. Actif en politique, il est présent dans les médias et populaire dans la communauté chiite dont il se pose comme le défenseur. Muhammad Mahdî Sams al-Dîn est son concurrent direct : en tant que président du Conseil supérieur chiite, instance créée à l'instigation de Mûsâ Sadr en 1967, il est le représentant officiel de la communauté chiite au Liban et participe en tant que tel aux débats politiques qui animent le pays. Ses avis comptent d'autant plus que, dans un Liban en reconstruction, il a choisi d'oeuvrer non pas contre l'État mais avec l'État, et pour plus d'intégration de la communauté chiite au Liban.

20 Muhammad Husayn Fadl Allâh et Muhammad Mahdî Sams al-Dîn déploient tous les deux une activité d'enseignement religieux au Liban ; parallèlement, chacun s'est implanté à Sayyida Zaynab où il préside aux destinées d'une école et en assure le financement. Fadl Allâh a repris en 1992 la tête d'une école fondée deux ans plus tôt par un groupe de clercs $^{34}$ et il y vient chaque semaine. Par ailleurs et à l'occasion de ses visites hebdomadaires à Damas, il entretient des contacts réguliers avec les chiites installés en Syrie tout comme avec les fonctionnaires de l'État iranien et la communauté chiite syrienne. Muhammad Mahdî Sams al-Dîn est beaucoup moins présent physiquement, mais la petite école dont il a la charge, ouverte en 1994 par des clercs irakiens associés à des Syriens, est en quelque sorte jumelée à son école libanaise.

21 Ainsi retrouve-t-on, dans les hawza-s, les tendances et les courants politiques et religieux qui animent les cercles chiites de Sayyida Zaynab, avec leurs deux axes principaux, l'un iranien et l'autre irakien; ce qui reflète bien les divisions du monde chiite d'aujourd'hui. A ces deux-là se greffent des courants représentés par des marga'-s locaux qui entendent jouer un rôle à l'échelle du Moyen-Orient. A ces compétitions autour de "la gestion des biens de salut", il faut ajouter la concurrence quotidienne et prosaïque qui s'exerce entre les clercs chiites exilés en Syrie et qui sont totalement tributaires d'un généreux donateur.

22 Cependant, aucun de tous ces marga $a^{\text {-s }}$ ne réside sur place. Par ailleurs, les hawza-s de Sayyida Zaynab ne forment pas de mugtahid-s. En effet, elles ne sauraient réellement y prétendre, car il leur faudrait des enseignants et des étudiants de haut niveau capables d'animer les cours de baht al-hârig où l'aspirant à l'ijtihâd puisse faire montre de ses capacités et de son savoir, briller, s'imposer, etc. Ainsi, bien que l'on ne puisse nier la place nouvelle que Sayyida Zaynab occupe dans le monde chiite depuis quelques années, il ne faudrait pas surestimer ses capacités en tant que foyer de savoir ou comme centre religieux. 
23 Certains voudraient bien que Sayyida Zaynab apparaisse comme une alternative à Nagaf et à Qumm. Prétention qui est favorisée par une conjonction de facteurs politiques. Le premier est la tension qui règne en Irak et l'oppression dirigée contre les chiites des villes saintes qui ont poussé ces derniers à fuir leur pays, provoquant ainsi le déclin de Nagaf. Le deuxième est la volonté de l'Iran de contrôler et de superviser les activités religieuses chiites à l'extérieur de ses frontières afin d'assurer son hégémonie sur tout le monde chiite duodécimain. Une volonté qui conduit l'Iran d'une part, à développer ses relations avec son seul allié arabe, la Syrie, ainsi qu'à consolider sa politique d'envoi de pèlerins dans ce pays ${ }^{35}$ et, d'autre part, à s'implanter dans les cercles chiites de Sayyida Zaynab. Enfin, tout cela est rendu possible grâce à la bienveillance des autorités syriennes. Les hawza-s, contrairement aux autres écoles religieuses du pays, ne disposent pas d'autorisation officielle du ministère des waqfs, hormis al-Hawza al-Zaynabiyya : même si les autorités l'ont exigé officiellement, les administrateurs des écoles ne semblent pas avoir jugé nécessaire d'en faire la demande. Ce qui ne les empêche pas de les faire fonctionner, profitant des franchises dont jouit Sayyida Zaynab. Mais rien n'étant officiel, l'État syrien reste seul maître du jeu, accordant ou non ses faveurs, et il n'est pas certain qu'il laisse les hawza-s se multiplier à l'avenir; sa position sur la question sera liée aux nouveaux développements de sa politique régionale.

24 Par ailleurs, Ridâ Murtadâ, l'administrateur du mausolée, s'oppose à la prolifération de ces écoles religieuses, trop traditionnelles à son goût ${ }^{36}$. De son côté, il a ouvert, fin avril 1995, un centre de documentation et de recherche, l'"Académie Sayyida Zaynab", situé dans l'enceinte du mausolée, destiné à des chercheurs et des conférenciers triés sur le volet. Nous avons donc à Sayyida Zaynab deux conceptions qui coexistent : d'un côté, celle de clercs non syriens, souvent militants d'un islam chiite engagé dans les affaires politiques; de l'autre, celle de notables locaux, garants de l'ordre établi, qui assurent le relais entre la communauté chiite imâmite syrienne et l'État.

Très minoritaires en Syrie, les duodécimains sont cependant les adeptes de ce que l'on qualifiera ici de doctrine chiite orthodoxe ${ }^{37}$. De leur côté, les ismaéliens, les druzes ou les alaouites sont bien souvent considérés comme des gulât, c'est-à-dire des chiites qui exagèrent leur culte de 'Alî et, à ce titre, peuvent se voir dénier la qualité de musulmans. De sorte que les contacts qu'entretiennent les lettrés, les intellectuels, les nationalistes et les 'ulamâ'-s chiites duodécimains et alaouites depuis le début du siècle ont servi à construire une alliance entre les deux communautés qui fut profitable à l'une comme à l'autre. Forts de cette alliance qui leur a permis de revendiquer la dignité de ga ${ }^{4}$ farites $^{38}$, les alaouites ont pu commencer à chercher la reconnaissance de leur islamité auprès de diverses autorités islamiques, sunnites et surtout chiites, au gré des opportunités et des nécessités politiques ${ }^{39}$. Les duodécimains de Syrie, eux, n'ont pu que se féliciter d'être en quelque sorte les garants d'une communauté confessionnelle dont les membres ont un accès privilégié aux rouages de l'État. Si les chiites au MoyenOrient sont en général dans l'opposition, se disant, selon leur terminologie consacrée, mazlûm-s (opprimés) - c'est le cas en Irak et dans une certaine mesure au Liban, ainsi que dans les pays du Golfe - ils sont dans une situation plutôt enviable en Syrie. Ce ne sont pourtant pas eux qui sont intéressés à faire de Sayyida Zaynab un grand centre religieux chiite : ils n'ont ni la force que confère une religion officielle, comme c'est le cas en Iran, ni le poids d'une communauté majoritaire comme c'est le cas des chiites irakiens. 


\section{NOTES}

1. Bâsil al-Asad s'est tué le 21 janvier 1994.

2. L'administrateur du waqf de Sayyida Zaynab ne dispose pas de chiffres permettant d'évaluer le pourcentage de chiites et de sunnites fréquentant le mausolée. Il remarque cependant que ce sont les chiites qui lui font des dons (entretien avec Ridâ Murtadâ, 13 septembre 1994).

3. Selon les vicissitudes politiques du moment, les villes saintes d'Irak, renfermant des tombeaux d'imâms, ne sont pas toujours accessibles aux Iraniens; par ailleurs, la tension qui règne aujourd'hui dans le sud de l'Irak décourage les pèlerins de s'y rendre. 4. Ali Shariati (m.1977) est le principal idéologue laïque de la révolution iranienne. Il prônait un islam progressiste.

5. Ibn Jobaïr, Voyages, traduction de Maurice Gaudefroy-Demombynes, 3ème partie, Paul Geuthner, Paris, 1953-1956, 409 p., p. 323 et p. 325.

6. Albert Hourani, "From Jabal 'Amil to Persia", Bulletin of the School of Oriental and African Studies, 49/1 (1986), pp. 133-140., p.133.

7. Cité par Henri Sauvaire, Description de Damas, Journal Asiatique, mai-juin 1896, p. 413, note 46.

8. Cf. Henri Sauvaire, op. cit., p. 387 et p. 453.

9. 'Abd al-Ganî al-Nâbulsî, Al-haqîqa wa al-magâz, al-hay'a al-misriyya al-'âmma li-lkitâb, Le Caire, 1986, p.215 et p. 245. Nous n'entrerons pas ici dans les débats entre savants musulmans sur l'identité de la femme enterrée à Râwiya et sur le lieu de la tombe de Zaynab, fille de 'Alî.

10. Le commandant Malinjoud décrivit ces cérémonies au début des années 20, dans, Revue d'histoire des religions, $\mathrm{n}^{\circ} 88$ (1923), pp. 165-172. Nous décrivons des séances de commémoration du martyre de Husayn tenues aujourd'hui par des chiites irakiennes dans "Ya Zaynab! Pratiques cultuelles chiites autour du mausolée de sayyida Zaynab, à Damas", à paraître dans Peuples Méditerranéens.

11. Sur les visites pieuses aux mausolées des imâm-s, cf. Ytzhak Nakash : "The Visitation of the Shrines of the Imams and the Shi'i Mujtahids in the Early Twentieth Century", SI, 81 (1995), pp. 153-164.

12. Voir la liste de ces mausolées dans Irene Calzoni, Shiite Mausoleums in Syria with Particular Reference to Sayyida Zaynab's Mausoleum", in La Shî́a nell'Impero ottomano, Conférence de Rome, 15/4/1991, Accademia Nazionale dei Lincei, Fondazione Leone Caetani, Rome, 1993, 224 p., pp. 191-201. Pour Alep, se reporter également à Jean Sauvaget, "Deux sanctuaires chiites d'Alep", Syria, 9 (1928), pp. 224-237 et à Biancamaria Scarcia Amoretti, "A proposito della tipologia religiosa sciita : AleppoMashad", in Studi in onore di Francesco Gabrieli, Université Sapienza, Rome, 1984, vol. II, 850 p., pp. 701-709.

13. Cf. Joseph Bahout, , Les Cahiers du CERMOC, n 7, Beyrouth, 1994, 154 p., pp. 52-54.

14. Village chiite situé dans la région d'Idlib, en Syrie.

15. Cf. Muhammad Fahrî Zakariyâ, Mintaqat al-sayyida zaynab bi-l-sâm, in al-Mawsim, vol. $1, \mathrm{n}^{\circ} 4,1989$, p. 888.

16. C'est une estimation. Ridâ Murtadâ, dans un document inédit sur Sayyida Zaynab, avance le chiffre de 200000 habitants, ce qui nous paraît excessif. Les statistiques officielles pour 1981 avançaient le chiffre de 31745 habitants. Selon le géographe Frank 
Meyer, les réfugiés du Golan forment aujourd'hui 50\% de la population; puis viennent les Palestiniens et les Dôm et Turkmân, des "gitans" sédentarisés qui y avaient acheté des terres bon marché dans les années 70. Mais cet auteur ne prend pas les ressortissants non syriens en compte. Cf. Frank Meyer, Dôm und Turkmân in Stadt und Land Damaskus, Erlanger Geographische Arbeiten, Sonderband 22, Erlangen, 1994, p. 66. 17. Le marga' est la référence à imiter en matière de précepte religieux; c'est le chef spirituel de la communauté. $C f$. $\mathrm{EI}^{2}$, vol. VI, pp. 533-541 l'article de Jean Calmard, Mardja'-i al-taqlîd.

18. Entretien avec Nasîb Murtadâ qui était membre fondateur de ce comité, Baalbek, 3 mai 1994.

19. Document inédit fourni par Ridâ Murtadâ. 7

20. Le legs pieux de terrains et de jardins par Husayn b. Mûsâ b. 'Alî al-Husaynî al-sâfi'î, mufti de la ville de Baalbek, ancêtre de la famille, date de $768 \mathrm{~h}$ (1367). Le texte de la waqfiyya est reproduit dans la thèse d'Irene Calzoni, Sayyida Zaynab. Notizie testimonianze dati sul suo mausoleo a Damasco, sous la direction de Giovanni Canova, Università degli studi di Venezia, 1988, 145 p. Cf. également la revue al-Mawsim, n52 (1996), pp. 16-29. 21. Le mujtahid est un clerc de haut niveau, capable d'interpréter la Loi divine. 22. Cf. sayyid 'Abd Allâh al-Hâsimî, âyat Allâh sayyid Hasan al-Sîrâzî, Qumm, s.d., 565 p., pp. 168-172.

23. Les quelques données et remarques qui suivent sur les hawza-s de Sayyida Zaynab sont le fruit d'une enquête sur ce sujet réalisée en collaboration avec Alfonso Ruiz, qui prépare un doctorat à l'Université autonome de Madrid, Religion y societad en Siria : el pueblo-mausoleo de Sayyida Zaynab. Cette enquête fera l'objet d'une prochaine publication commune.

24. Le wakîl est une sorte de délégué du marja dans un pays ou une région : il perçoit notamment les impôts canoniques et les redistribue au nom du marja` et suivant ses directives.

25. Dans l'enquête citée plus haut, la direction de l'école n'a pas répondu aux questions relatives à son mode d'administration ni à son financement. Bien qu'elle ne se prononce pas non plus sur la possibilité d'obtenir une carte de séjour pour ses étudiants, il semble que cela soit le cas et qu'elle jouisse d'excellentes relations avec les autorités syriennes. Ce qui n'est pas le cas des écoles plus récentes, qui ne peuvent procurer un titre de résidence à leurs étudiants non syriens.

26. Sur le système traditionnel de l'enseignement religieux chiite, $c f$. notre article, "La quête du savoir à Najaf. Les études religieuses chez les chi' ites imâmites de la fin du XIX ${ }^{\mathrm{e}}$ siècle à 1960", Studia Islamica, 81(1995), pp. 165-185.

27. C'est évidemment une description idéale du système. Cependant, nous approuvons Michael Fischer lorsqu'il estime que, plus ou moins, la réalité correspond à cet idéal. $C f$. Iran from Religious Dispute to Revolution, Cambridge-Londres, Harvard University Press, 1980, 314 p., p. 63.

28. Voir à ce sujet 'Alî al-Bahâdilî, Al-hawza al-'ilmiyya fí al-Nagaf. Ma'âlimuhâ wa harakatuhâ al-islâhiyya, 1920-1980, dâr al-Zahrâ', Beyrouth, 1993, 480 p.

29. 'Alî al-Bahâdilî, Wamdât min hayât al-imâm al-Khû'î, Dâr al-qârî, Beyrouth, 1992, 163 p., p. 5.

30. Sur le principe de la marga'iyya, cf. Jean Calmard, "Mardja'i taklîd", EI² vol. VI, pp. 533-541 et sur la "crise" que traverse cette institution depuis la disparition de Khû'î, voir Pierre Martin, "La direction religieuse chiite dans une zone de turbulences", Peuples Méditerranéens, n64-65 (1993), pp. 241-264. 
31. Nous ne disposons pas de chiffre concernant ces étudiants étrangers, ni d'indication sur la durée de leurs séjours.

32. C'est une marga'iyya sinon officielle et approuvée par des textes de loi, au moins officieuse et qui s'affiche en public. Lors du décès de Bâsil al-Asad, un encart bien visible a été publié dans la presse syrienne, présentant les condoléances du "marga". 33. Il faut resituer ici le terme "marga ‘iyya locale" dans la géographie des communautés chiites. Il s'oppose au terme "marga'iyya supérieure" (a'lâ) supposée recouvrir tout le monde chiite.

34. Il ne l'a donc pas fondée lui-même, contrairement à ce qu'indique un article paru sur cette école, "Hawzat al-Murtadâ fî baldat al-Sayyida Zaynab (Sûriyya)", al-Mawsim, $\mathrm{n}^{\circ} 23-24$ (1995), p. 523.

35. Sur l'histoire contemporaine des relations entre l'Iran et la Syrie, $c f$. Shireen T. Hunter, "Iran and Syria : From Hostility to Limited Alliance" in Amirahmadi H. et Entessar N. éd., Iran and the Arab World, pp. 198-216.

36. Cela n'empêche pas la présence d'étudiants et d'enseignants chiites syriens dans les écoles de Sayyida Zaynab, sans compter quelques syriens d'autres sectes chiites, alaouites, druzes et ismaéliens.

37. La question de l'orthodoxie en matière de chiisme serait à développer. Nous nous bornerons ici à expliquer cette position par : le poids démographique des duodécimains, leur importance politique (notamment par le fait que leur religion est la religion de l'État en Iran), l'existence d'une tradition savante non ésotérique, donc accessible; enfin, paradoxalement, leur reconnaissance, par les sunnites, en tant que musulmans.

38. Le mot ga'farite vient du nom du sixième imâm Ga'far al-Sâdiq qui, selon la tradition chiite, fonda une école juridique. Il renvoie donc à cette école à laquelle se rattache les chiites duodécimains, c'est-à-dire à un fiqh élaboré, enseigné et reconnu.

39. Consulter à ce sujet l'article de Martin Kramer, "Syria's Alawis and Shi'ism", in Shi'ism, Resistance and Revolution, Martin Kramer éd., New York, Westview Press, 1987, 324 p., pp. 237-254.

\section{RÉSUMÉS}

Sayyida Zaynab reçoit chaque année la visite de plus d'un million de pélerins qui vont pleurer les ahl al-bayt, les membres de la famille du Prophète. Depuis la fin du siècle dernier, le pèlerinage se "chiitise », ce qui rend la ville doublement étrangère aux yeux des Syriens. Les raisons en tiennent, d'une part, au fait que les pratiques culturelles chiites sont distinctes des sunnites et, d'autre part, au fait que la plupart des pélerins sont des Iraniens, c'est-à-dire des populations non arabes. 